Review Article

\title{
The Biochemistry, Toxicology, and Uses of the Pharmacologically Active Phytochemicals: Alkaloids, Terpenes, Polyphenols, and Glycosides
}

\author{
Chinaza Godswill Awuchi \\ Department of Physical Sciences, Kampala International University, Kampala, Uganda \\ *Corresponding author: Chinaza Godswill Awuchi I Email: awuchichinaza@gmail.com
}

Received: 7 Oktober 2019; Revised: 7 November 2019; Accepted: 17 November 2019; Published: 6 December 2019

\begin{abstract}
The research focused on the biochemistry and the medicinal uses of alkaloids, terpenes, polyphenols, and glycosides. Several parts of plants such as bark, root, leaf, stem, etc. are being used to revert abnormalities back to normal, prevent illnesses, or allay symptoms. These plant parts contain bioactive compounds such as terpenes, glycosides, polyphenols, and alkaloids. Alkaloids have a range of pharmacological activities such as antiasthma (e.g. ephedrine), anticancer (e.g. homoharringtonine), antimalarial (e.g. quinine). Other alkaloids possess stimulant (e.g. cocaine, caffeine, theobromine, nicotine) and psychotropic activities (e.g. psilocin), and have been widely used as recreational drugs or in entheogenic rituals. Alkaloids can be toxic too (e.g. tubocurarine, atropine). Although alkaloids act on diverse metabolic systems in humans and animals, they almost uniformly induce a bitter taste. The characteristics and number of the structures of phenol in polyphenols underlie the unique chemical, physical, and biological (metabolic, therapeutic, toxic, etc.) properties of specific members of the class; e.g. ellagitannin and tannic acid. Generally, foods often contain complex mixtures of polyphenols. Several polyphenolic extracts, such as from grape seeds, olive pulp, maritime pine bark, or grape skin, are sold as ingredients for functional foods, cosmetics, and dietary supplements. Terpenoids and terpenes are the primary components of the essential oils of most types of flowers plants. Essential oils are used widely as scents and fragrances in perfumery and also in traditional medicine, such as aromatherapy. Similarly, many plant glycosides are used as medicines. Amygdalin and laetrile (a synthetic derivative) were investigated as potential drugs for cancer treatment and were promoted as alternative medicine; nevertheless, they are ineffective and dangerous. Pharmacologically active phytochemicals can have useful applications, as well as safety concerns.
\end{abstract}

Keywords: medicinal plants, phytochemicals, alkaloids, terpenes, polyphenols, glycosides

\section{INTRODUCTION}

The use of plants to cure many human diseases has a long history. Several parts of plants such as the leaf, stem, bark, root, among others, are being used to prevent, allay symptoms or to revert abnormalities back to normal. As the practices of herbal remedies do not strictly adhere to facts accrued with scientific approaches, orthodox medicine perceives herbal medicines as alternative medicines. However, most pharmaceutical products presently dispensed by physicians and pharmacists have a history of usage as herbal remedies, including digitalis, quinine, opium, and aspirin. Modern medicine today uses active compounds isolated from the higher plants, and about 80 percent of these active ingredients show positive correlation between their traditional uses and the modern therapeutic uses (1). 
The use of, and search for dietary supplements and drugs obtained from plants have increased recently. Scientists and researchers such as botanists, phytochemists, pharmacologists, microbiologists, and food chemists are exploring the Earth for phytochemicals and the clues that can be developed into medicines for the treatment of various diseases. Traditional medicine is still recognized and accepted as the desired primary health care system in several communities, with over $80 \%$ in developing countries and about $60 \%$ of the world's population directly depending on the medicinal plants for their medical treatment and health purposes (2). Most natural occurring sugar alcohols can be used for medicinal purposes, especially as laxatives $(3,4)$.

Currently, millions of people all-over the world consume plant-based medications as part of the traditional medicine for a wide range of medical disorders. The utilization of traditional medicine in developing and underdeveloped countries directly contributes to the socio-economic status, wellbeing, and needs of the rural communities $(5,6)$. People in rural areas generate income from medicinal plants, especially herbalists and traditional healers. The use of medicinal plants particularly in primary health care system has become prominent, and has led to the growing search for plants with medicinal application and use. The search and quest for plants with medicinal uses have led to many ethnobotanical studies that documented species of traditional medicinal plant, the preparation mode, and uses by local communities in many parts of the country $(7,8)$. The identifications of these medicinal plants and in later stage the investigations of the quality and toxicity are of the utmost importance. Moreover, a lot of invaluable indigenous information about the uses of medicinal plants are being lost from one generation to the other and with the growing rate of destruction of habitats, plant resources including the medicinal plants are threatened or are getting depleted.

\section{DISCUSSION}

\subsection{Alkaloids}

Alkaloids are class of organic compounds that occur naturally and mostly contain basic atoms of nitrogen. Also, this group includes various related compounds with weak acidic and neutral properties. Some synthetic compounds of similar structure can also be termed alkaloids (9-10). Along with carbon, hydrogen and nitrogen, alkaloids can also contain sulfur, oxygen, and, often rarely, other elements such as phosphorus, chlorine, and bromine. Morphine, the first individual alkaloid, was isolated from the opium poppy (Papaver somniferum) in 1804 (11).

Alkaloids are produced by a wide range of organisms including fungi, bacteria, plants, and animals (12). They are usually purified from the crude extracts of these organisms through acid-base extractions, or solvent extraction followed by silica-gel column chromatography (13). Alkaloids have various pharmacological activities such as antimalarial (e.g. quinine), anticancer (e.g. homoharringtonine), antiasthma (e.g. ephedrine) (14), cholinomimetic (e.g. galantamine) (15), vasodilatory (e.g. vincamine), analgesic (e.g. morphine), antiarrhythmic (e.g. quinidine), antihyperglycemic activities (e.g. piperine), and antibacterial (e.g. chelerythrine). Many have found use and applications in modern or traditional medicine, or as the starting points for drug discovery. Many other alkaloids possess stimulant (e.g. cocaine, caffeine, theobromine, nicotine) and psychotropic activities (e.g. psilocin), and have been used as recreational drugs or in entheogenic rituals. Alkaloids can be toxic too (e.g. tubocurarine, atropine) (16). Although alkaloids act on a variety of metabolic systems in humans and in other animals, they nearly uniformly evoke a bitter taste (9).

Boundary between alkaloids and the other nitrogen-containing natural compounds is ambiguous. Compounds like proteins, amino acid peptides, nucleotides, nucleic acid, antibiotics, and amines, are usually not referred to as alkaloids. The natural compounds with nitrogen in the exocyclic position (dopamine, mescaline, serotonin, etc.) are often classified as amines than as alkaloids (17). Some authors and researchers, however, consider alkaloids special case of amines (18). 


\subsubsection{Classifications of alkaloids}

There is no uniform classification. In the beginning, when there was lacking of knowledge of chemical structures, botanical classifications of the source plants were greatly relied on. Currently, this classification is considered obsolete. Compared with many other classes of natural compounds, the alkaloids are characterized by great structural diversity. More recent classification is based on the similarity of the carbon skeleton (e.g., isoquinoline-, pyridine-, indole-like) or biochemical precursor (lysine, tyrosine, tryptophan, ornithine, etc.). Nevertheless, they require compromises in the borderline cases; e.g., nicotine contains a pyrrolidine part from ornithine and a pyridine fragment from nicotinamide and as a result can be assigned to both classes.

The alkaloids are usually divided into the following main grouping:

a) True alkaloids; which contain nitrogen $(\mathrm{N})$ in the heterocycle and have their source from amino acids. Characteristic examples are nicotine, morphine, and atropine. Also, this group includes few alkaloids that besides the nitrogen heterocycle have peptide (e.g. ergotamine) or terpene fragments (e.g., evonine). The piperidine alkaloids coniceine and coniine may be considered as true alkaloids (instead of pseudoalkaloids, as can be seen below) though they do not originate from the amino acids.

b) Polyamine alkaloids - derivatives of spermidine, spermine, and putrescine.

c) Protoalkaloids, which contain nitrogen (not the nitrogen heterocycle) and originate from amino acids. Examples are mescaline, ephedrine, and adrenaline.

d) Pseudoalkaloids - alkaloid-like compounds which do not originate from the amino acids. This group includes steroid-like and terpene-like alkaloids, and also purine-like alkaloids such as theacrine, theophylline, caffeine, and theobromine. Some authors and researchers classify as pseudoalkaloids such compounds such as cathinone and ephedrine. Those originate from amino acid phenylalanine, but acquire their nitrogen atom through transamination, not from the amino acid.

e) Peptide and cyclopeptide alkaloids.

Some alkaloids do not have carbon skeleton characteristic of the group they belong. Homoaporphines and galanthamine do not contain isoquinoline fragment, but they are generally attributed to the isoquinoline alkaloids.

\subsubsection{Properties of alkaloids}

Most alkaloids have oxygen in their molecular structure; the compounds are often colorless crystals at ambient conditions. The oxygen-free alkaloids, such as coniine or nicotine, are typically colorless, oily, volatile liquids. Several alkaloids are colored, like sanguinarine (orange) and berberine (yellow). Bufotenin contains an indole core and can be produced from the amino acid tryptophan in living organisms. The nicotine molecule contains both pyrrilidine and pyridine rings.

Most alkaloids are weak base, but some, such as theophylline and theobromine, are amphoteric (19). Many alkaloids poorly dissolve in water but dissolve readily in organic solvents, such as chloroform, 1,2-dichloroethane, or diethyl ether. Caffeine, nicotine, cocaine, and codeine are slightly soluble in water (with solubility of $\geq 1$ g per liter), while others, including yohimbine and morphine, are very slightly soluble in water $(0.1-1 \mathrm{~g} / \mathrm{L})$. Acids and alkaloids form salts of various strengths. The salts are often freely soluble in ethanol and water and poorly soluble in many organic solvents. A few exceptions include the water-soluble quinine sulfate, and the scopolamine hydrobromide, that is soluble in organic solvents.

Many alkaloids have a bitter taste and poisonous when consumed. Alkaloid production in plants seemed to have evolved in natural response to feeding by herbivorous animals; although, some animals 
have acquired the ability to detoxify alkaloids. Many alkaloids may produce developmental defects in the offsprings of animals that ingest but cannot detoxify alkaloids. A typical example is the alkaloid cyclopamine, which is produced in the leaves of the corn lily. In 1950s, about $25 \%$ of lambs born by the sheep that grazed on corn lily had severe facial deformations. These deformations ranged from deformed jaws to cyclopia. After decades of research, in 1980s, the compound 11-deoxyjervine (an alkaloid), later renamed to cyclopamine, was identified as been responsible for these deformities (20).

\subsubsection{Distribution of alkaloids in nature}

Generally, alkaloids are generated by several living organisms, especially by the higher plants about $10-25 \%$ of those contain alkaloids. Thus, earlier the term "alkaloid" was linked with plants. The alkaloids in plants is often within a few percent and is also inhomogeneous over the tissues of plants. Depending on type of plants, maximum concentration is often observed in the fruits or seeds (Strychnine tree), leaves (black henbane), bark (cinchona), or root (Rauwolfia serpentina). Furthermore, different tissues of same plants can contain different alkaloids.

Outside plants, alkaloids are also found in animals, such as bufotenin in the skin of many toads, in many insects, markedly ants, and in some types of fungi, such as psilocybin in fungus of the genus Psilocybe (21). Also, many marine organisms contain alkaloids. Several amines, such as serotonin and adrenaline, which play a significant role in the higher animals, are similar to the alkaloids in their biosynthesis and structure and are sometimes referred to as alkaloids.

\subsubsection{Alkaloids Extraction}

Due to the alkaloid's structural diversity, there is no single method extracting from natural raw materials. Majority of the methods exploit the properties of most alkaloids, especially solubility in organic solvents but not in water, as well as the opposite tendency of their salts.

Many plants contain numerous alkaloids. Their mixture is first extracted and then the individual alkaloids are separated. The plants are ground thoroughly before the extraction. Most alkaloids exist in the raw plants in form of salts of the organic acids. The extracted alkaloids can remain salts or may change into bases. The base extraction is accomplished by processing the raw materials with alkaline solutions and extracting the alkaloids bases with organic solvents, such as chloroform, diethyl ether, benzene, or 1,2dichloroethane. The impurities are then dissolved by weak acids; which converts the alkaloid bases to salts that are washed with water. If required, an aqueous solution of the alkaloid salts is once again made alkaline and can be treated with organic solvent. The process is done repeatedly until the desirable purity is achieved.

In acidic extraction, the raw plant materials are processed by weak acidic solution (for example, acetic acid in water, methanol, or ethanol). A base is then added to transform the alkaloids to basic forms which are extracted with organic solvents (if the extraction was done with alcohol, first it is removed, and the remainder dissolved in water). In addition, the solution is purified as explained above. Alkaloids are usually separated from their mixtures using their different solubility in some solvents and different reactivity with some reagents or by distillation process.

A number of alkaloids have been identified from insects, among which the alkaloids, known as solenopsins, from fire ant venom have received greater attention from researchers (22). These insect alkaloids can be extracted efficiently by centrifugation of live ants or by solvent immersion of the live fire ants (23) followed by purification using silica-gel chromatography. Dosing and tracking the extracted solenopsin has been described as likely based on their absorbance peak near $232 \mathrm{~nm}$ (24). Crystals of piperine are extracted from the black pepper.

\subsubsection{Biosynthesis of alkaloids}

Biological precursors of many alkaloids are amino acids, such as lysine, phenylalanine, aspartic acid, anthranilic acid, tyrosine, tryptophan, ornithine, and histidine. Nicotinic acid can be synthesized from 
aspartic acid or tryptophan. The ways of alkaloid biosynthesis are too many and cannot be classified easily. However, there are a few distinctive reactions involved in the biosynthesis of many classes of alkaloids, including Mannich reaction and synthesis of Schiff bases.

Mannich reaction

An integral constituent of the Mannich reaction, along with a carbonyl compound and an amine, is a carbanion that plays the role of nucleophile in the nucleophilic addition to ion formed by the reaction of the carbonyl and the amine.

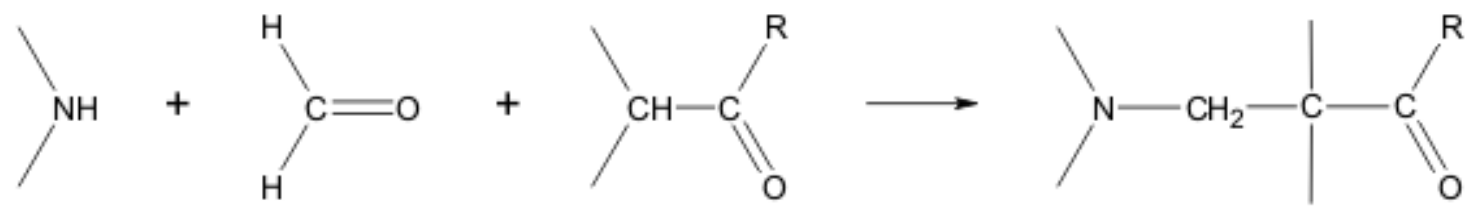

The Mannich reaction can proceed both intramolecularly and intermolecularly:

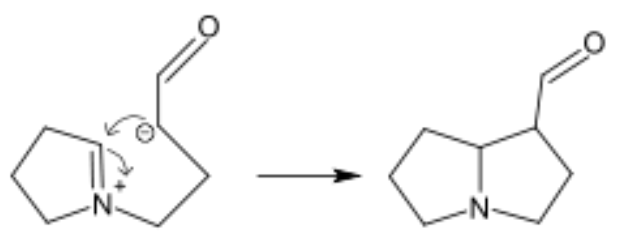

Synthesis of Schiff bases

The Schiff bases can be synthesized by reacting amines with aldehydes or ketones. These reactions are common methods of producing $\mathrm{C}=\mathrm{N}$ bonds.

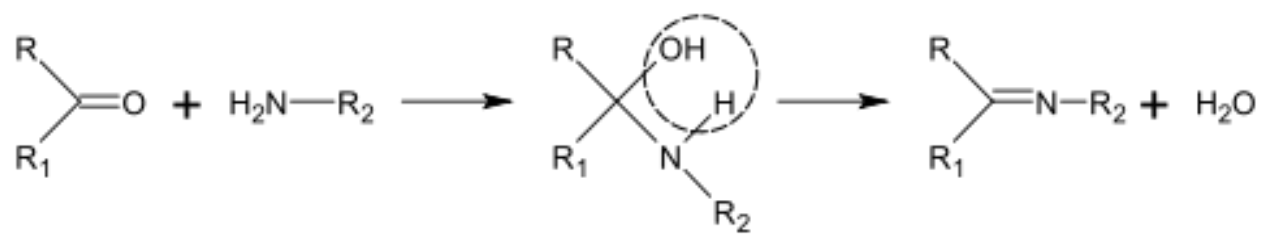

In biosynthesis of alkaloids, such reactions can take place in a molecule, for example, in the synthesis of piperidine:

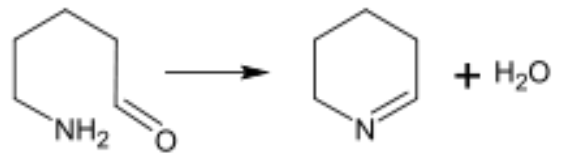

\section{Dimer alkaloids}

In addition to the above described monomeric alkaloids, there are also dimeric, trimeric and tetrameric alkaloids formed by the condensation of two, three, or four monomeric alkaloids. The dimeric alkaloids are often formed from the monomers of the same type by the following mechanisms:

- Mannich reaction, resulting in, e.g., voacamine

- Condensation of the aldehydes with amines (toxiferine)

- Michael reaction (villalstonine)

- Lactonization (carpaine).

- Oxidative addition of phenols (tubocurarine, dauricine) 
There are also dimeric alkaloids produced from two distinctive monomers, such as the vinca alkaloids vincristine and vinblastine, which are made from the coupling of vindoline and catharanthine $(25,26)$. Vinorelbine, a newer semi-synthetic chemotherapeutic agent, is used in treatment of the non-small-cell lung cancer (27). It is another derivative dimer of catharanthine and vindoline and is synthesized from anhydrovinblastine (28) starting either from leurosine (29) or the monomers themselves.

\subsubsection{The biological roles of alkaloids}

The role of alkaloids for the living organisms that produce them is not clear. Initially it was presumed that the alkaloids are the end products of the metabolism of nitrogen in plants, as urea in mammals. Later it was shown that the concentration of alkaloids varies with time, and this hypothesis was contradicted. Also, many ants are suggested to produce alkaloids as components of their venom, however the exact pathways for the biosynthesis have not been empirically established (30).

Most of the functions of alkaloids already identified are related to protection. For example, liriodenine, aporphine alkaloid, produced by tulip tree protects the tree from parasitic mushrooms. Additionally, the presence of alkaloids in plant prevents chordate animals and insects from eating it. Plants use alkaloids as part of their defense mechanisms against insects, chordate animals, etc. However, some animals are adaptive to alkaloids and can use them in their own metabolism. Some alkaloid-related substances such as serotonin, histamine, and dopamine are important neurotransmitters in animals. Many alkaloids are also reported to regulate plant growth. One typical example of an organism that make use of alkaloids for protection is the Utetheisa ornatrix, commonly known as ornate moth. The pyrrolizidine alkaloids render the larvae and adult moths unpalatable to lots of their natural enemies such as insectivorous hemiptera, insectivorous bats, coccinelid beetles, and green lacewings (31). Another example of alkaloids in use occurs in Agonopterix alstroemeriana (the poison hemlock moth). The moth feeds on its alkaloid-rich and highly toxic host plant poison hemlock (Conium maculatum) in its larval stage. Agonopterix asltroemeriana may benefit twofold from toxicity of naturally-occurring alkaloids, both through the ability of Agonopterix alstroemeriana to recognize the Conium maculatum as the correct location for the oviposition, and through the non-palatability of the species to predators (32). Solenopsin, a fire ant venom alkaloid, has been shown to protect queens of the invasive fire ants during foundation of new nests, therefore playing a significant role in spread of this species of pest ant around the world (33).

\subsubsection{Applications and uses of alkaloids}

Use as psychoactive drug: preparations of plants containing alkaloids and their extracts, and later the pure alkaloids, have long been applied and used as psychoactive substances. Caffeine, cathinone, and cocaine are the CNS (central nervous system) stimulants. Mescaline and various indole alkaloids (such as dimethyltryptamine, ibogaine, and psilocybin) have hallucinogenic effect. Codeine and morphine are strong narcotic pain killers.

Also, there are alkaloids which do not have strong psychoactive effects themselves, but are the precursors for semi-synthetic psychoactive drugs, e.g., pseudoephedrine and ephedrine are used to produce methamphetamine and methcathinone. Thebaine is used in synthesis of several painkillers such as oxycodone.

Use in medicine: the medical use of plants containing alkaloid has a long history, and, as a result, when the first set of alkaloids were isolated in $19^{\text {th }}$ century, they instantly found application and use in clinical practice. Several alkaloids are still used in medicine, usually in the form of salts, as shown in Table 1. 
Table 1. Alkaloids and their use in medicine

\begin{tabular}{ll}
\hline Alkaloid & Action \\
\hline Ajmaline & antiarrhythmic \\
Atropine, hyoscyamine, scopolamine & anticholinergic \\
Caffeine & adenosine receptor antagonist, stimulant \\
Codeine & analgesic, antitussive \\
Colchicine & remedy for gout \\
Emetine & emesis, antiprotozoal agent \\
Ergot alkaloids & vasoconstriction, Uterotonic, hallucinogenic \\
Morphine & analgesic \\
Nicotine & nicotinic acetylcholine receptor agonist, stimulant \\
Physostigmine & acetylcholinesterase inhibitor \\
Quinidine & antiarrhythmic \\
Quinine & antimalarial, antipyretic \\
Reserpine & antihypertensive \\
Tubocurarine & relax muscle \\
Vinblastine, vincristine & antitumor \\
Vincamine & antihypertensive, vasodilating \\
Yohimbine & aphrodisiac, stimulant \\
\hline
\end{tabular}

Many semisynthetic and synthetic drugs (medicines) are structural modifications of alkaloids, which were designed to change or enhance the primary effect of the drug and reduce undesirable sideeffects. For example, an opioid receptor antagonist called naloxone is a derivative of thebaine which is present in the opium.

Use in agriculture: Prior to development of various relatively low-toxic synthetic pesticides, many alkaloids, such as salts of anabasine and nicotine, were used as insecticides. Their high toxicity to humans limited their use (34).

\subsection{Polyphenols}

Polyphenols also called polyhydroxyphenols are a structural class of mostly natural, but also semisynthetic or synthetic, organic chemicals characterized by presence of huge multiples of phenol structural units $(35,36)$ characteristics and number of the structures of these phenol underlie the unique chemical, physical, and biological (therapeutic, metabolic, toxic, etc.) properties of specific members of the class. Some examples are ellagitannin and tannic acid (tannin). The historically significant chemical class of the tannins is subset of the polyphenols (35). Plant-derived polyphenol, tannic acid (tannin), is formed by the esterification of ten equivalents of phenylpropanoid-derived gallic acid to glucose (a monosaccharide) core from primary metabolism. Phenol-phenolate resonance structures and equilibrium giving rise to phenol aromatic reactivity.

Individual polyphenols engage in the reactions associated to both their core phenol structures, their linkages, and the types of glycosides they form. The standard phenolic reactions are oxidations to para- and ortho-quinones (which contributes to antioxidant characteristics), underlying aromatic transformations related to presence of the phenolic hydroxyl, and ionization (which contributes to complexation and solubility); reactions related to their linkages include oxidative and hydrolytic bond cleavages, and nucleophilic additions. Additionally, a traditional feature of the polyphenols was their ability to form specific, characteristic metal complexes (37).

\subsubsection{Structural features of polyphenols}

Opposite to the smaller phenols, the polyphenols are often macromolecules (larger molecules) deposited in the cell vacuoles. The upper limit of molecular weight for small molecules is 800 Daltons, 
which makes allowance for the possibility of rapid diffusion across cell membranes so they can reach the intracellular sites of action or can remain as pigments once cell senesces. Hence, numerous larger polyphenols are biosynthesized in-situ from the smaller polyphenols to non-hydrolysable tannins and remain undiscovered and unidentified in the plant matrix. Most polyphenols have repeating phenolic moieties of resorcinol, pyrogallol, phloroglucinol, and pyrocatechol linked by more stable C-C bonds (nonhydrolysable condensed tannins) or esters (hydrolysable tannins). Proanthocyanidins are mostly polymeric units of epicatechin and catechin. Resorcinol (1,3-benzenediol) and catechol types of polyphenols have two, and phloroglucinol (benzenetriol) and pyrogallol types have three phenol hydroxyl groups, respectively, although mixing of these types in polyphenols is also likely. The phenolic substructures arise from many biosynthetic pathways (WBSSH definition), especially polyketide and phenylpropanoid branches aimed at plant and the related secondary metabolites. The C-glucoside substructure of polyphenols is typified by puerarin (the phenol-saccharide conjugate), a mid-molecularweight natural plant product. The phenol attachment to the saccharide is through a carbon-carbon bond (C-C). The isoflavone and benzopyran (10-atom "fused ring" system of isoflavone), also a structural feature here, is commonly found in polyphenols.

Other than hydroxyl groups, polyphenols often have heteroatom substituents; ester and ether linkages are common, as are many carboxylic acid derivatives; ester linkages are common in the hydrolysable tannins. In addition to the simple heteroatom links, the carbon frameworks may become complex, e.g., several C-C linkages join hydrolytically labile ethers and esters as common in nonhydrolysable tannins. The biaryl/biphenyl substructure of polyphenols can be prepared by synthesis using the copper-mediated Ullmann reaction. Also, the $\mathrm{C}-\mathrm{C}$ bond in biaryls in nature is synthesized through a metal-mediated coupling reaction, always involving iron. The biaryl substructure is found in ellagic acid. Because of the preponderance of the saccharide-derived core structures, as well as spiro- and other structure types, natural chiral (stereo) centers abound.

\subsubsection{Chemical synthesis of polyphenols}

True polyphenols from tannin and other WBSSH types are consistently biosynthesized in the natural sources where they derive; their chemical syntheses (with standard bench organic chemical methods) were to some extent limited until first decade of the new millennium due to these syntheses involve challenging stereoselectivity and regioselectivity issues (38). Early work focused on achiral synthesis of phenolic-related constituents of polyphenols in the late 1970's, and the Nelson \& Meyers synthesis of permethyled derivative of the ubiquitous and abundant diphenic acid core of the ellagitannins in 1994 (39) followed by the stereoselective synthesis of the more complex permethylated structures such as Itoh and coworker's synthesis of permethylated pedunculagin with specific attention to the axial symmetry issues in 1996, and Lipshutz and coworkers' (+)-tellimagrandin II derivative in 1994 (40). The total synthesis of a completely unmasked polyphenol that of ellagitannin tellimagrandin I, was diastereoselective sequence reported by Feldman et al (41). Further total syntheses of the deprotected polyphenols that followed were headed by the Feldman group, for example in Feldman and Lawlor's synthesis of ellagitannin, coriariin A and other tannin relations (42). Khanbabaee and Grosser achieved a relatively efficient total synthesis of the pedunculagin in $2003(43,44)$.

Work proceeded with a focus on enantioselective total syntheses, for example, on atroposelective syntheses of the axially chiral biaryl polyphenols $(45,46)$ with recent further significant work including the controlled assembly of various polyphenols according to integrated strategies, like in the syntheses of extended series of procyanidins (an oligomeric catechins) by many groups and of resveratrol polyphenols by Snyder group at Columbia which included the diverse ampelopsins $\mathrm{G}$ and $\mathrm{H}$, carasiphenols $\mathrm{B}$ and $\mathrm{C}$, and nepalensinol B (47). A biomimetic synthesis, as well as the first formal total synthesis of the 5-ODesgalloyl-epi-punicacortein A, an additional ellagitannin in its C-glucosyl (the C-glucoside subclass), has 
recently been accomplished also. The novel strategies and methods mentioned in these recent examples helped in opening the field of the polyphenol chemical synthesis to such an unprecedented degree (48).

\subsubsection{Chemical properties of polyphenols}

Polyphenols are molecules owing their Ultraviolet/Visible absorptivity to aromatic structures with great conjugated systems of pi electron configurations; also, they have autofluorescence properties, particularly lignin and the phenolic part of the suberin. They are reactive species to oxidation. ABTS may be used to characterize polyphenol oxidation products (49). Polyphenols also characteristically possess an important binding affinity for proteins, which may lead to the formation of the soluble and the insoluble protein-polyphenol complexes (50).

\subsubsection{Chemical uses of polyphenols}

Traditionally, some polyphenols are used as dyes. For example, in the Indian subcontinent, the peel of pomegranate, high in tannins and other polyphenols, or the pomegranate juice, is employed in dyeing of non-synthetic fabrics (51), especially tannins, were traditionally used for tanning leather and are currently used as precursors in green chemistry especially to produce plastics or resins by polymerization with or without use of formaldehyde or the adhesives for particleboards. Generally, the objectives are to make use of pecan shells left after processing or the plant residues from grape, olive (known as pomaces) (52). Pyrocatechin and pyrogallol are among oldest photographic developers.

\subsubsection{The biological role in plants}

Both the larger polyphenols play important roles in the ecology of many plants (9). Their known effects in the tissues of plants can be divided into following categories (53):

- UV screens to provide coloration (plant pigments) and to protect against ionizing radiation.

- Signaling molecules in growth processes, including ripening.

- Release and suppression of the growth hormones such as auxin.

- Prevention of microbial infections (phytoalexins).

- Deterrence of herbivores (sensory properties).

\subsubsection{Polyphenols occurrence in nature}

The most abundant polyphenols in nature are the condensed tannins, which are found in virtually all plants families. Larger polyphenols are always concentrated in bark layers, flowers, fruits, the leaf tissue, and the epidermis but also play significant roles in nutrient cycles in forest ecology, and the decomposition of forest litter. Absolute concentrations of the total phenols in plant tissues widely differ depending on the type of polyphenols, assay, and literature source; they range from 1 to $25 \%$ total polyphenols and natural phenols, calculated with reference to dry green leaf mass (54).

High levels of polyphenols in various woods can explain their natural preservations against rot. Flax and a submerged aquatic plant called Myriophyllum spicatum secrete polyphenols which are involved in allelopathic interactions (55). Also, polyphenols are found in animals. In some arthropods such as crustaceans and insects polyphenols play a role in sclerotization (epicuticle hardening). The cuticle hardening is due to the presence of the enzyme polyphenol oxidase. Apparently, there is no polyphenol tanning occurring in the arachnids' cuticle. In crustaceans, there is second oxidase activity resulting in cuticle pigmentation.

\subsubsection{Biosynthesis and metabolism of polyphenols}

Polyphenols incorporate smaller parts (components) and building blocks from the simpler natural phenols that originate from the phenyl propanoid pathway for phenolic acids or shikimic acid pathway for the gallotannins and analogs. Caffeic acid derivatives and flavonoids are biosynthesized from malonylCoA and phenyl alanine. Complex gallotannins develop through dimerization processes resulting in hydrolyzable tannins or through in-vitro oxidation of 1,2,3,4,6-pentagalloyl-glucose. For anthocyanidins, 
the precursors of condensed tannin biosynthesis, leucoanthocyanidin reductase (LAR) and dihydroflavonol reductase are crucial enzymes with the subsequent addition of epicatechin and catechin moieties for larger, non-hydrolysable tannins (56). The glycosylated form develops from the glucosyltransferase activity and increases the polyphenols solubility (57).

Polyphenol oxidase (PPO) is an enzyme which catalyzes the oxidation of o-diphenols to give oquinones. It is the rapid polymerization of o-quinones to produce red, black, or brown polyphenolic pigments that is responsible for browning in fruits, such as browning in apple when cut or bruised. In insects, polyphenol oxidase serves for the hardening of the cuticle. Laccase is a major enzyme which initiates the cleavage of hydrocarbon rings that catalyze the addition of a hydroxyl group (-OH) to phenolic compounds. The enzyme can be found in fungi such as Panellus stipticus, organisms that break down the lignin, a complex polymer (aromatic) in the wood that is highly resistant to the degradation by conventional and normal enzyme systems. Hypericin, phenolic lipids, and anthracyclines are derived from polyketides cyclisation (58).

\subsubsection{Polyphenols content in food}

Generally, foods contain complex polyphenols mixtures. The most important food sources are the commodities commonly consumed in large quantities such as green tea, black tea, fruit and vegetables, red wine, coffee, extra virgin olive oil, chocolate, and olives. Herbs and spices, algae and nuts are also potentially significant for supplying some polyphenols. Many polyphenols are specific to particular foods (phloridzin in apples, isoflavones in soya, flavanones in citrus fruit); while others, such as quercetin, are detected in all the plant products such as vegetables, cereals, leguminous plants, fruits, tea, and wine (59).

Some polyphenols are considered anti-nutrients, substances that interfere with absorption of essential nutrients, particularly iron and other metal ions, and also by binding to the digestive enzymes and other proteins, especially in ruminants (60). However, a few polyphenols have antioxidant properties. The phenolic and the carotenoid compounds with antioxidant properties in the vegetables have been found to be significantly retained better by steaming than by frying. Polyphenols in beer, wine, and numerous nonalcoholic juice beverages could be removed by using finings, substances that are often added near or at the completion of the brewing processing.

\subsubsection{Potential health effects of polyphenols}

Many polyphenolic extracts from sources, such as grape seeds, grape skin, maritime pine bark, and olive pulp, are sold as ingredients in cosmetics, functional foods, and dietary supplements with little or no proof of effect or legal health claims (61). Some polyphenol ingredients have self-aclaimed GRAS status in the US.

The diverse structures and indeterminate metabolic fate of the phenolic compounds following digestion prevent the understanding about their potential health effects. Especially, due to the presumed antioxidant role of the polyphenols in vivo cannot be well established, the US FDA issued labeling direction to manufacturers that the polyphenols cannot be declared as antioxidant nutrients except physiological evidence exists to confirm such a qualification and also a DRI value has been established $(61,62)$. Furthermore, since the purported health claims for some specific polyphenol-enriched foods remain unproven (63) health statements regarding the polyphenols on product labels are banned by the EFSA and the FDA (64). However, the EFSA recently recognized health claims of some specific products, such as olive oil and cocoa (65).

Compared with the in vitro effects of polyphenols, the possible in vivo functions remain unknown due to (a) long-term studies failing to show effects with an action mechanism, sensitivity and efficacy or specificity (61); (b) the absence of any validated in vivo biomarkers; and (c) invalid applications of high, non-physiological test concentrations in in vitro studies, which are subsequently not relevant for design of in vivo experiments. 
2.2.10. Use of polyphenols in traditional medicine

Herbal teas contain soluble polyphenols that impart astringent effects believed to have medicinal properties (66). In Ayurveda system of folk medicine, pomegranate and its polyphenol-rich peel are thought to be useful for therapeutic purposes.

\subsubsection{Sensory properties of polyphenols}

With respect to food and beverages, the main cause of astringency is not entirely understood, but it is biochemically measured as the ability of a substance or compound to precipitate proteins. A review article published in 2005 found that bitterness decreases and astringency increases with mean degree of polymerization. For the water-soluble polyphenols, the molecular weights between $500-3000$ were reported to be necessary for protein precipitation. However, the smaller molecules might still have some astringent qualities possibly due to formation of non-precipitated complexes with proteins or the crosslinking of proteins with the simple phenols that have 1,2,3-trihydroxy or 1,2-dihydroxy groups (67)). Also, flavonoid configurations can cause significant differences in sensory properties, for example, epicatechin is more astringent and bitter than its chiral isomer catechin. By contrast, hydroxycinnamic acids do not have any astringent qualities, but are bitter (68).

2.2.12. Extraction of polyphenols

Extraction of polyphenols can be done using a solvent like methanol, water, hot water, methanol/formic acid, water/methanol/acetic or formic acid etc. The extraction by liquid-liquid or countercurrent chromatography can also be done. Solid phase extraction can also be done on C18 sorbent cartridges. Some other techniques are heat reflux extraction, ultrasonic extraction, microwaveassisted extraction (69), use of ethanol in immersion extractor, or critical carbon dioxide, pressurized liquid extraction. The extraction conditions (temperature, solvent and concentrations, ratio of solvent to raw material, extraction time) have to be optimized.

Mainly found in the fruit seeds and skins, high levels of polyphenols may only reflect the EPP (measured extractable polyphenol) content of a fruit which also may contain non-extractable polyphenols. The black tea contains high quantities of polyphenol which makes up $20 \%$ of its weight (70) Purification can be accomplished by preparative chromatography. Concentration can be done by ultrafiltration.

\subsubsection{Analytical techniques}

The analysis techniques are those of the phytochemistry: including extraction, isolation, structural elucidation, and then quantification (71). Phosphomolybdic acid is used as reagent for staining phenolics in TLC (thin layer chromatography). Polyphenols may be studied by spectroscopy, particularly within the ultraviolet domain, by paper or fractionation chromatography. They can also be analyzed by chemical characterization.

Instrumental chemistry analyses include separation by HPLC (high performance liquid chromatography), and especially by RPLC (reversed-phase liquid chromatography), can be coupled to mass spectrometry (MS). Purified compounds can be identified by means of nuclear magnetic resonance (NMR). The DMACA reagent is a histological dye specific to the polyphenols used in microscopy analyses. Also, auto-fluorescence of polyphenols can be used, especially for localization of suberin and lignin.

Polyphenol content can be quantified isolation/separation by volumetric titration. Permanganate, an oxidizing agent, is used to oxidize known concentration of a standard solution of tannin, producing a standard curve. Tannin content of the unknown (sample) is then expressed as equivalents of appropriate condensed or hydrolysable tannin. Some methods for the quantification of total content of polyphenol are based on colorimetric measurements. Various tests are relatively specific to polyphenols (e.g. the Porter's assay). The total phenols (or the antioxidant effect) can be measured with the Folin-Ciocalteu reaction. The 
results are typically expressed as the gallic acid equivalents. The polyphenols are rarely evaluated by antibody technologies.

Some other tests measure a fraction's antioxidant capacity. Some make use of ABTS radical cation which is very reactive to most antioxidants including thiols, phenolics, and vitamin $C$ (72). During this reaction, blue ABTS radical cation is transformed back to its initial colorless neutral form. The reaction can be spectrophotometrically monitored. This assay is often called the TEAC (Trolox equivalent antioxidant capacity) assay. The reactivity of the numerous tested antioxidants are always compared to that of Trolox, a vitamin $\mathrm{E}$ analog.

The other antioxidant capacity assays that use Trolox as standard include oxygen radical absorbance capacity (ORAC), diphenylpicrylhydrazyl (DPPH), ferric reducing ability of plasma (FRAP) (73) assays or inhibition of the copper-catalyzed in vitro human oxidation of low-density lipoprotein. New methods including use of biosensors can assist in monitoring the content of polyphenols in food (74). Quantitative results produced by the mean of the diode array detector-coupled high performance liquid chromatography are in general given as relative rather than the absolute values as there is lack of commercially available standards for entire polyphenol molecules.

\subsection{Terpenes}

Terpenes are a large, diverse class of organic compounds, made by various plants, especially conifers, and by some insects $(75,76)$. Terpenes are hydrocarbons. They often have a strong odour and may protect plants that produce them by attracting predators and the parasites of herbivores, and by deterring herbivores (77, 78). Although occasionally used interchangeably with the terpenes, the terpenoids (or isoprenoids) are the modified terpenes as they have additional functional groups, often oxygen-containing.

Terpenes are the major constituents of turpentine and of rosin produced from resin. Terpenes are also main biosynthetic building blocks. For example, steroids are derivatives of triterpene squalene. Terpenes and terpenoids are the primary components of the essential oils of various types of flowers and plants. Essential oils are widely used as fragrances in traditional medicine and perfumery, such as aromatherapy. Vitamin A is a terpenoid. The synthetic variations and the derivatives of natural terpenes and terpenoids greatly expand the range of flavors used in food additives and aromas used in perfumery.

\subsubsection{Structure and biosynthesis}

Terpenes are biosynthetically derived from units of isopentenyl pyrophosphate. Although the structures of terpenoids are reorganized as derivatives of isoprene (i.e. 2-methyl-1,3-butadiene), the isoprene is not involved in the terpenoids biosynthesis. The $C_{5}$ rule or the biogenetic isoprene rule was described by Leopold Ružička (in 1953), who explained that terpinoids can be pictured as the result of linking units of isoprene head-to-tail to form chains that can be arranged to give rings.

There are two metabolic pathways that create terpenoids: the Mevalonic acid pathway and the 2C-methyl-D-erythritol 4-phosphate/1-deoxy-D-xylulose 5-phosphate (MEP/DOXP) pathway. Many organisms produce terpenoids through the pathway of HMG-CoA reductase, called the Mevalonate pathway, which produces cholesterol too. One of the intermediate compounds and substances in this pathway is the mevalonic acid. The range of reactions take place in cytosol. The MEP/DOXP pathway, also called mevalonic acid-independent pathway or non-mevalonate pathway, takes place in the apicomplexan protozoa and plastids of plants, as well as in several bacteria.

Geranyl pyrophosphate is converted to geranylgeranyl pyrophosphate and farnesyl pyrophosphate, respectively C20 and C15 precursors to diterpenes and sesquiterpenes (as well as diterpenoids and sesequiterpenoids). The biosynthesis is mediated by terpene synthase $(79,80)$. 


\subsubsection{Types of terpenes}

Terpenes may be classified according to the number of units of isoprene in the molecule; prefix in the name specifies the number of terpene units required to assemble the molecule.

a) Hemiterpenes are made of $a$ single isoprene unit. Isoprene is considered the only hemiterpene; oxygen-containing derivatives such as isovaleric acid and prenol are hemiterpenoids.

b) Monoterpenes consist of two units of isoprene and have the general molecular formula $\mathrm{C}_{10} \mathrm{H}_{16}$. The examples of monoterpenes and monoterpenoids are terpineol (in lilacs), geraniol, limonene (in citrus fruits), linalool (in lavender), pinene (in pine trees), and myrcene (in hops), (81).

c) Sesquiterpenes (The sesqui- prefix means one and a half) consist of three units of isoprene and have the general molecular formula $\mathrm{C}_{15} \mathrm{H}_{24}$. The examples of sesquiterpenes and sesquiterpenoids are farnesol, humulene, farnesenes, etc.

d) Diterpenes consist of four units of isoprene and have the general molecular formula $\mathrm{C}_{20} \mathrm{H}_{32}$. They are derived from geranylgeranyl pyrophosphate. Typical examples of diterpenes and diterpenoids are kahweol, cembrene, taxadiene (precursor of taxol), and cafestol. Diterpenes also form basis for important biological compounds such as phytol, retinol, and retinal.

e) Sesterterpenes are terpenes having 25 carbons and five units of isoprene and are rare relative to the other sizes. An example of sesterterpenoid is geranylfarnesol. The sester- prefix means two and a half.

f) Triterpenes have the molecular formula $\mathrm{C}_{30} \mathrm{H}_{48}$ and consist of six isoprene units. The linear triterpene squalene, major shark liver oil constituent, is derived from reductive coupling of two farnesyl pyrophosphate molecules. Squalene is then biosynthetically processed to generate either cycloartenol or lanosterol, the structural precursors to all steroids.

g) Sesquarterpenes have the molecular formula $\mathrm{C}_{35} \mathrm{H}_{56}$ and are composed of seven isoprene units. Sesquarterpenes are typically microbial in origin. Examples of sesquarterpenoids are tetraprenylcurcumene and ferrugicadiol.

h) Tetraterpenes contain eight units of isoprene and have the general molecular formula $\mathrm{C}_{40} \mathrm{H}_{64}$. Important biological tetraterpenoids include the monocyclic gamma-carotene, the bicyclic betaand alpha-carotenes, and the acyclic lycopene.

i) Polyterpenes consist of long chains of several isoprene units. Some plants produce a polyisoprene with the trans double bonds, called gutta-percha. Natural rubber consists of polyisoprene with the cis double bonds.

j) Norisoprenoids, such as $C_{13}$-norisoprenoids 3-oxo- $\alpha$-ionol found in Muscat of Alexandria leaves and the 7,8-dihydroionone derivatives, such as 3-oxo-7,8-dihydro- $\alpha$-ionol and megastigmane-3,9diol found in wine (responsible for some spice notes in Chardonnay) or the Shiraz leaves (both grapes in species Vitis vinifera), can be produced by fungal peroxidases (82) or glycosidases.

\subsubsection{Properties and uses of terpenes}

Terpenes have many desirable properties for use in pharmaceutical, food, cosmetics, biotechnology industries (83). Terpenes are valuable active ingredients as part of the natural agricultural pesticides. They are used by the termites of subfamily Nasutitermitinae to ward off predators such as insects, through use of specialized mechanism known as "fontanellar gun". Higher quantities of terpenes are released from trees in warmer weather, and can be a natural form of the cloud seeding. The cloud reflects sunlight, allowing forest temperature to regulate (84). The flavor and aroma of hops comes partly from sesquiterpenes (mainly $\beta$-caryophyllene and $\alpha$-humulene), which affect beer quality (85). The genomes of species of 17 plants contain genes that encode the terpenoid synthase enzymes imparting the terpenes with their basic structure, and the cytochrome P450s which modify this basic structure (86). Terpenes are also major components of Cannabis sativa plants, which have at least 120 identified 
compounds $(87,88)$. Terpenes have gained public awareness by the growth and education of recreational and medical cannabis. Companies and organizations operating in the cannabis markets have pushed marketing and education of terpenes in their products as a major way to differentiate the taste and effects of cannabis. Entourage Effect, which explains the synergy of terpenes, cannabinoids, and other compounds in plants, has also helped create further awareness and raise the demand for terpenes in the products of cannabis.

\subsubsection{Industrial syntheses}

While terpenes and terpenoids widely occur, their extraction from natural sources is always challenging. Consequently, they are often produced from petrochemicals by chemical synthesis. In one route, acetylene and acetone are condensed to yield 2-Methylbut-3-yn-2-ol, which is then extended with the acetoacetic ester to yield geranyl alcohol. Many others are prepared from those terpenoids and terpenes which are readily isolated in amount, say from the paper and the tall oil industries. For instance, $\alpha$-pinene, which is readily obtained from natural sources, is converted to camphor and citronellal. Citronellal is also converted to menthol and rose oxide (75).

\subsection{Glycosides}

A glycoside (in chemistry) is a molecule with a sugar bound to another functional group through a glycosidic bond. Glycosides play many important roles in living organisms. Several plants store chemicals in form of inactive glycosides, which may be activated by enzyme hydrolysis (89) causing the sugar part to break off, resulting in the availability of chemical for use. Lots of such plant glycosides are used as medicines. Many species of Heliconius butterfly have the capability to incorporate these plant compounds as form of chemical defense against their predators. In humans and animals, poisons are often bound to molecules of sugar as part of their elimination mechanisms from the body. Amygdalin was the first glycoside ever identified, by the French chemists Antoine Boutron-Charlard and Pierre Robiquet, in 1830.

Any molecule with a sugar group bonded via its anomeric carbon to another group through a glycosidic bond is a glycoside. Glycosides can be linked by an S-(a thioglycoside), C- (a C-glycoside), O(an O-glycoside), or N- (a glycosylamine) glycosidic bond. According to IUPAC, the preferred term is the Cglycosyl compound; the name $C$-glycoside is a misnomer (90). The given definition is used by IUPAC, which also recommends the Haworth projection to appropriately assign stereochemical configurations (91). Many authors require in addition that sugar be bonded to non-sugar for a molecule to qualify as a glycoside, thereby excluding polysaccharides. Then the sugar group is known as the glycone and the nonsugar group known as the genin or aglycone part of the glycoside. The glycone may consist of monosaccharide (a single sugar group), disaccharide (two sugar groups), or oligosaccharide (several sugar groups).

\subsubsection{Gycosides related compounds}

Molecules containing N-glycosidic bond are called glycosylamines. Many biochemistry authors call these compounds $\mathrm{N}$-glycosides and place them in the group of glycosides; which is considered a misnomer and strictly discouraged by the IUPAC. Glycosylamines and glycosides are together grouped as glycoconjugates; other glycoconjugates include peptidoglycans, glycolipids, lipopolysaccharides, glycoproteins, and glycopeptides.

\subsubsection{Chemistry of glycosides}

The glycone and aglycone parts can be separated chemically by hydrolysis in presence of acid and can also be hydrolyzed by alkali. Also, there are many enzymes that form and break glycosidic bonds. Most significant synthetic enzymes in nature are the glycosyltransferases, and the most significant cleavage 
enzymes are glycoside hydrolases. Glycosynthases (genetically altered enzymes termed) have been developed to form glycosidic bonds in outstanding yield.

There are many ways glycosidic bonds can be chemically synthesized. The Koenigs-Knorr reaction involves the condensation of glycosyl halides and the alcohols in presence of metal salts such as mercuric oxide or silver carbonate. Fischer glycosidation refers to synthesis of glycosides by reaction of unprotected monosaccharides with the alcohols (often as solvent) in presence of strong acid catalyst.

\subsubsection{Classification of glycosides}

Glycosides can be classified by the type of glycosidic bond, by the glycone, and by the aglycone.

a. By type of glycosidic bond

Dependent on whether the glycosidic bond lies above or below the plane of the cyclic molecule of sugar, glycosides are classified as $\beta$-glycosides or $\alpha$-glycosides. Some enzymes such as the $\alpha$-amylase can hydrolyze $\alpha$-linkages alone; others, such as the emulsin, can only affect $\beta$-linkages.

The Four type of linkages present between the glycone and the aglycone are:

- N-linkage/glycosidic bond

- O-linkage/glycosidic bond

- C-linkage/glycosidic bond, nonhydrolysable by enzymes or acids

- S-linkage/glycosidic bond

b. By glycone (presence of sugar)

If glycone group of any glycoside is glucose, the molecule is referred to as a glucoside; if it is fructose, the molecule is referred to as a fructoside; if it is glucuronic acid, the molecule is referred to as a glucuronide; etc. In the body, toxic substances are usually bonded to glucuronic acid to increase their solubility in water; then the resulting glucuronides are excreted.

c. By aglycone

Glycosides can also be classified according to the aglycone chemical nature. For purposes of pharmacology, food chemistry, and biochemistry, this is the major useful classification.

Alcoholic glycosides; Example of alcoholic glycoside is salicin found in the genus Salix. In the body, salicin can be converted into salicylic acid, which is closely related to the aspirin and has antipyretic, antiinflammatory, and analgesic effects.

Steviol glycosides; these glycosides have the steviol as the aglycone part. Steviol glycosides are sweet glycosides in the stevia plant Stevia rebaudiana Bertoni. They have 40 to 300 times the sweetness of sucrose. Stevioside and rebaudioside A, the two primary glycosides, are used as natural sweeteners in some countries. Glucose or combinations of rhamnose-glucose are bound to the aglycone ends to form the different compounds.

Anthraquinone glycosides; these group of glycosides contain an aglycone group which is a derivative of anthraquinone. Anthraquinone glycosides have a laxative effect, and are mainly found in the dicot plants except the Liliaceae family which are monocots. Anthranol and anthron are reduced forms of anthraquinone. Anthraquinone glycosides are present in rhubarb, senna, and Aloe species.

Cardiac glycosides or steroidal glycosides; here the aglycone part is steroidal nucleus. They are found in the plant genera Scilla, Strophanthus, and Digitalis. They are used in treatment of heart diseases, for example, congestive heart failure (historically as now recognized does not improve survivability; other agents are currently preferred) and arrhythmia.

Thioglycosides; these compounds contain sulfur; as the name implies (q.v. thio-). Examples are sinalbin, found in white mustard, and sinigrin, found in black mustard. 
Iridoid glycosides; these contain iridoid group; e.g. theviridoside, Loganin, aucubin, Geniposidic acid, and Catalpol.

Coumarin glycosides; the aglycone is either coumarin or a derivative. Example is apterin which has been be shown to dilate the coronary arteries and block calcium channels. Some coumarin glycosides are obtained from the dried leaves of the Psoralea corylifolia. Chromone glycosides; the aglycone, in this case, is called benzo-gamma-pyrone. Cyanogenic glycosides; in cyanogenic glycosides, the aglycone contains a cyanohydrin group. The plants that make the cyanogenic glycosides store them in the plant vacuole, but, if the plant is under attack, they are released and activated by enzymes in cytoplasm. These do away with the sugar part of the molecule, causing the cyanohydrin structure to collapse, resulting in the release of toxic hydrogen cyanide $(\mathrm{HCN})$. Storing them in their inactive forms in the plant vacuole prevents them from damaging and harming the plant under normal conditions (92).

Along with playing an important role in deterring herbivores, in many plants they control carbon and nitrogen transport, germination, bud formation, and possibly act as antioxidants. The making of cyanogenic glycosides is a conserved function of evolutionary, appearing in the species as old as the ferns and as recent as the angiosperms. These compounds are produced by about 3,000 species; in the screens they are found in approximately 11 percent of cultivated plants but only 5 percent of plants overall-humans appear to have selected for them (92).

Examples include prunasin and amygdalin which are produced by the bitter almond tree; other plant species that produce cyanogenic glycosides are barley, flax, sorghum (in which dhurrin, first cyanogenic glycoside to be officially identified, was first isolated), white clover (Gleadow and Møller, 2014), and cassava (which makes linamarin, iso-linamarin, and lotaustralin). Amygdalin and a synthetic derivative known as laetrile were examined as potential drugs for cancer treatment and were promoted heavily as alternative medicine; however, they are ineffective and dangerous (93). Some species of butterfly, such as Parnassius smintheus and Dryas iulia, have evolved to use cyanogenic glycosides in their host plants for protection against predators via their unpalatability.

Flavonoid glycosides; the aglycone in the flavonoid glycosides is a flavonoid. The examples of this big group of glycosides include:

- Naringin (glycone: Rutinose, aglycone: Naringenin)

- Hesperidin (glycone: Rutinose, aglycone: Hesperetin)

- Quercitrin (aglycone: Quercetin, glycone: Rhamnose)

- Rutin (aglycone: Quercetin, glycone: Rutinose)

They are also known to reduce capillary fragility. Among the important flavonoids effects are their antioxidant effect.

Phenolic glycosides; in this group, the aglycone is simple phenolic structure. Example is arbutin in Common Bearberry Arctostaphylos uva-ursi, which has urinary antiseptic effects.

Saponins; saponin glycosides are found in liquorice. They also cause the hemolysis of red blood cells. When shaken with water, the compounds give a permanent froth. Their medicinal value is due to their antiinflammatory, expectorant, and corticoid effects. Steroid saponins, e.g., in the Dioscorea wild yam sapogenin diosgenin-in the form of its glycoside dioscin-is an essential starting material for the production of semi-synthetic glucocorticoids and many other steroid hormones such as progesterone. Ginsenosides are the triterpene glycosides and the Ginseng saponins from Panax Ginseng A. C. Meyer, (Chinese ginseng) and the Panax quinquefolius (American Ginseng). Generally, in organic chemistry, the use of the word "saponin" is discouraged, because several plant components can produce foam, and various triterpene-glycosides are amphipolar in certain conditions, acting as a surfactant. Modern uses of saponins in biotechnological industries are as adjuvants in vaccines: the Quil A and its derivative QS-21 
which are isolated from the bark of the Quillaja saponaria Molina, to stimulate both Th1 immune response and production of CTLs (cytotoxic T-lymphocytes) against the exogenous antigens make them ideal for application and use in subunit vaccines and in the vaccines directed against intracellular pathogens and also for therapeutic cancer vaccines though with aforementioned side-effect of hemolysis (94). Also, saponins are natural ruminal antiprotozoal agents and are potential to improving the ruminal microbial fermentation, thus reducing methane production and ammonia concentrations in ruminant animals (95).

\section{CONCLUSION}

Various parts of plants such as leaf, stem, bark, root, etc. are being used to prevent, allay symptoms or revert abnormalities back to normal. These plants contain bioactive compounds such as terpenes, glycosides, polyphenols, and alkaloids. Alkaloids have a wide range of pharmacological activities including antimalarial (e.g. quinine), antiasthma (e.g. ephedrine), anticancer (e.g. homoharringtonine). Other alkaloids possess psychotropic (e.g. psilocin) and stimulant activities (e.g. cocaine, caffeine, nicotine, theobromine), and have been used in entheogenic rituals or as recreational drugs. Alkaloids can be toxic too (e.g. atropine, tubocurarine) (Robbers et al., 1996). Although alkaloids act on a diversity of metabolic systems in humans and other animals, they almost uniformly evoke a bitter taste. The number and characteristics of the phenol structures in polyphenols underlie the unique physical, chemical, and biological (metabolic, toxic, therapeutic, etc.) properties of particular members of the class; examples include tannic acid and ellagitannin. Generally, foods contain complex mixtures of polyphenols. Many polyphenolic extracts, such as from grape skin, grape seeds, olive pulp or maritime pine bark, are sold as ingredients in functional foods, dietary supplements, and cosmetics. Terpenes and terpenoids are the primary constituents of the essential oils of many types of plants and flowers. Essential oils are used widely as fragrances in perfumery and traditional medicine, such as aromatherapy. Many plant glycosides are used as medications. Amygdalin and a synthetic derivative, laetrile, were investigated as potential drugs to treat cancer and were heavily promoted as alternative medicine; however, they are ineffective and dangerous.

\section{References}

1. Sarkar, S.; Zaidi, S.; Chaturvedi, A.K.; Srivastava, R.; Dwivedi, P.K.; Shukla, R. Search for a herbal medicine: Antiasthmatic activity of methanolic extract of Curcuma longa. Journal of Pharmacognosy and Phytochemistry, 2015, 3, 59-72.

2. Shrestha, P.M.; Dhillion, S.S. Medicinal plant diversity and use in the highlands of Dolakha district, Nepal. Journal of Ethnopharmacology, 2003, 86(1), 81-96.

3. Awuchi, Chinaza, g.; Echeta, K.C. Current developments in sugar alcohols: Chemistry, nutrition, and health concerns of sorbitol, xylitol, glycerol, arabitol, inositol, maltitol, and lactitol. International Journal of Advanced Academic Research, 2019, 5(11), 1 - 33.

4. Awuchi, C.G. Sugar alcohols: Chemistry, production, health concerns and nutritional importance of mannitol, sorbitol, xylitol, and erythritol. International Journal of Advanced Academic Research, 2017, 3(2); 31 - 66.

5. Tabuti, J.R.S.; Dhillion, S.S.; Lye, K.A., Traditional medicine in Bulamogi County, Uganda. Its practitioners, users \& viability. Journal of Ethnopharmacology, 2003, 85, 119-129.

6. Chiranjibi, P.; Sudhakar, R.; Dhal, N.K.; Rashmita, D. Some phytotherapeutic claims by tribals of Rayagada district, Orissa, India. Ethnobotanical Leaflets, 2006, 10, 189-197.

7. Tabuti, J.R.S.; Kukunda, C.B.; Waako, W.J. Medicinal plants used by traditional medicine practitioners in the treatment of tuberculosis and related ailments in Uganda. Journal of Ethnopharmacology, 2010, 127, 130-136.

8. Lamorde, M.; Tabuti, J.R.S.; Obua, C.; Kukunda-Byobona, C.; Lanyero, H.; Byakika- Kibwika, P.; Bbosa, S.G.; Lubega, A.; Ogwal-Okeng, J.; Ryan, M.; Waako, P.J.; Merry, C. Medicinal plants used by traditional medicine 
practitioners in the treatment of HIV/AIDS and related conditions in Uganda. Journal of Ethnopharmacology, 2010, 130, 43-53..

9. Awuchi, Chinaza, G. Medicinal plants: The medical, food, and nutritional biochemistry and uses, International Journal of Advanced Academic Research, 2019, 5(11); 220 - 241.

10. Robert, A.L. Lewis' dictionary of toxicology. CRC Press, 1998, p. 51

11. Gansäuer, A.; Justicia, J.; Fan, C.A.; Worgull, D.; Piestert, F. Reductive $C-C$ bond formation after epoxide opening via electron transfer". In Krische, Michael J. (ed.). Metal Catalyzed Reductive C-C Bond Formation: A Departure from Preformed Organometallic Reagents. Topics in Current Chemistry. 279. Springer Science \& Business Media. pp. 25-52.

12. Roberts, M.F. 1998. Alkaloids: Biochemistry, Ecology, and Medicinal Applications. Wink, Michael. Boston, MA: Springer US. ISBN 9781475729054 . OCLC 851770197.

13. Gonçalves, P.F.; Eduardo; Russ-Solis; Daniel; Delazari, D.S.; Lucilene; Aparecido, D.S; Pinto, J.R.; Ribeiro, D.S.M,; Anally; Cardoso, M.C.S. 2013.

14. Kittakoop, P.; Mahidol, C.; Ruchirawat. S. Alkaloids as important scaffolds in therapeutic drugs for the treatments of cancer, tuberculosis, and smoking cessation. Curr Top Med Chem. 2014, 14 (2): $239-252$.

15. Russo, P.; Frustaci, A.; Del-Bufalo, A.; Fini, M.; Cesario, A. Multitarget drugs of plants origin acting on Alzheimer's disease. Curr Med Chem. 2013, 20 (13): 1686-93. doi:10.2174/0929867311320130008.

16. Robbers, J.E.; Speedie, K.M.; Tyler, V.E. Chapter 9: Alkaloids". Pharmacognosy and Pharmacobiotechnology. Philadelphia: Lippincott, Williams \& Wilkins. 1996. pp. 143-185. ISBN 978-0683085006.

17. Leland, J.C. Natural Products from Plants, Second Edition. CRC, 2006, p. 30 ISBN 0-8493-2976-0.

18. Raj, K.B. A Text Book of Organic Chemistry. 4th Edition, New Age International, 2004, p. 644 ISBN 81-224-14591.

19. Spiller G. A. Caffeine, CRC Press, 1997 ISBN 0-8493-2647-8.

20. Thomas, A.; Colin, S.S.; Pennycott, T.W. Poisonous plants and related toxins, Vol. 2001. CABI. 2004, p. 362. ISBN 978-0-85199-614-1.

21. Touchard, A.; Aili, S.; Fox, E.; Escoubas, P.; Orivel, J.; Nicholson, G.; Dejean, A. the biochemical toxin arsenal from ant venoms". Toxins, 2016, 8(1), 30. doi:10.3390/toxins8010030.

22. Fox, E.; Gonçalves, P. Gopalakrishnakone, P.; Calvete, Juan J. (eds.), "Venom Toxins of Fire Ants", Venom Genomics and Proteomics: Venom Genomics and Proteomics, Springer Netherlands, 2016, pp. 1-16, doi:10.1007/978-94-0076649-5_38-1

23. Fox, E.G.P.; Xu, M.; Wang, L.; Chen, L.; Lu, Y.Y. Speedy milking of fresh venom from aculeate hymenopterans". Toxicon, 2018, 146, 120-123. doi:10.1016/j.toxicon.2018.02.050.

24. Fox, E.G.P.; Xu, M.; Wang, L.; Chen, L.; Lu, Y.Y. Gas-chromatography and UV-spectroscopy of Hymenoptera venoms obtained by trivial centrifugation". Data in Brief. 2018, 18, 992-998. doi:10.1016/j.dib.2018.03.101.

25. Hirata, K.; Miyamoto, K.; Miura, Y. Catharanthus roseus L. (Periwinkle): Production of Vindoline and Catharanthine in Multiple Shoot Cultures". In Bajaj, Y. P. S. (ed.). Biotechnology in Agriculture and Forestry 26. Medicinal and Aromatic Plants. VI. Springer-Verlag. 1994, pp. 46-55.

26. Gansäuer, A.; Justicia, J.; Fan, C.A.; Worgull, D.; Piestert, F. Reductive C-C bond formation after epoxide opening via electron transfer". In Krische, Michael J. (ed.). Metal Catalyzed Reductive C-C Bond Formation: A Departure from Preformed Organometallic Reagents. Topics in Current Chemistry. 279. Springer Science E Business Media. 2007, pp. 25-52. doi:10.1007/128_2007_130.

27. Faller, B.A.; Pandi, T.N. Safety and efficacy of vinorelbine in the treatment of non-small cell lung cancer". Clinical Medicine Insights: Oncology, 2011, 5, 131-144. doi:10.4137/CMO.S5074.

28. Morcillo, S.P.; Miguel, D.; Campaña, A.G.; Cienfuegos, L.A.D.; Justicia, J.; Cuerva, J.M. Recent applications of $\mathrm{Cp}_{2} \mathrm{TiCl}$ in natural product synthesis. Organic Chemistry Frontiers, 2014, 1(1), 15-33. doi:10.1039/c3qo00024a.

29. Ngo, Q.A.; Roussi, F.; Cormier, A.; Thoret, S.; Knossow, M.; Guénard, D.; Guéritte, F. Synthesis and biological evaluation of Vinca alkaloids and phomopsin hybrids. Journal of Medicinal Chemistry, 2009, 52(1): 134142. doi:10.1021/jm801064y.

30. Pianaro, A.; Fox, E.G.P.; Bueno, O.C.; Marsaioli, A.J. Rapid configuration analysis of the solenopsins. Tetrahedron: Asymmetry, 2012, 23 (9), 635-642. 
31. Conner W.E. Tiger Moths and Woolly Bears - behaviour, ecology, and evolution of the Arctiidae. New York: Oxford University Press. 2009, pp. 1-10.

32. Castells, E.; Berenbaum, M.R. Laboratory rearing of agonopterix alstroemeriana, the defoliating poison hemlock (Conium maculatum 1.) moth, and effects of piperidine alkaloids on preference and performance. Environmental Entomology, 2006, 35 (3), 607-615. doi:10.1603/0046-225x-35.3.607

33. Fox, E.G.P.; Wu, X.; Wang, L.; Chen, L.; Lu, Y.Y.; Xu, Y. Queen venom isosolenopsin A delivers rapid incapacitation of fire ant competitors. Toxicon, 2019, 158, 77-83.

34. György, M.; Miklós, N.; Viktor, A. Pesticide chemistry, Elsevier, 2002, pp. 21-22.

35. Quideau, S.P.; Deffieux, D.; Douat-Casassus, C. L.; Pouységu, L. Plant polyphenols: chemical properties, biological activities, and synthesis. Angewandte Chemie International Edition, 2011,50(3), 586621. doi:10.1002/anie.201000044.

36. Quideau, S. Why bother with polyphenols?. Groupe Polyphenols. Retrieved 26 March 2014.

37. Haslam, E.; Cai, Y. Plant polyphenols (vegetable tannins): Gallic acid metabolism. Natural Product Reports, 1994, 11(1): 41-66. doi:10.1039/NP9941100041.

38. Krohn, K.; Ahmed, I.; John, M.; Letzel, M. C.; Kuck, D. Stereoselective Synthesis of Benzylated Prodelphinidins and Their Diastereomers with Use of the Mitsunobu Reaction in the Preparation of Their Gallocatechin Precursors". Eur. J. Org. Chem. 2010, 13, 2544-2554.

39. Nelson, T.D.; Meyers, A.I. A rapid total synthesis of an ellagitannin (sic). J. Org. Chem. 1994, 59(9), 25772580. doi:10.1021/jo00088a046.

40. Itoh, T.; Chika, J.; Shirakami, S. Synthesis of trideca-O-methyl-alpha-pedunculagin. Diastereo-favoritism studies on intramolecular ester-cyclization of axially chiral diphenic acids with carbohydrate core. J. Org. Chem. 1996, 61(11): 3700-3705. doi:10.1021/jo950969j.

41. Feldman, K.S.; Ensel, S.M. Ellagitannin chemistry. Preparative and mechanistic studies of the biomimetic oxidative coupling of galloyl esters. J. Am. Chem. Soc. 1994, 116(8), 3357-3366. .

42. Feldman, K.S.; Lawlor, D.M.; Sahasrabudhe, K. Ellagitannin chemistry. Evolution of a three-component coupling strategy for the synthesis of the dimeric ellagitannin coriariin $\mathrm{A}$ and a dimeric gallotannin analogue". J. Org. Chem. 2000, 65(23): 8011-8019. doi:10.1021/jo0010936.

43. Khanbabaee, K.; Grosser, M. An efficient total synthesis of pedunculagin by using a twofold intramolecular double esterification strategy. Eur. J. Org. Chem. 2003, 11, 2128-2131.

44. Feldman, K.S. Recent progress in ellagitannin chemistry. Phytochemistry. 2004, 66(17), 1984-2000.

45. Bringmann, G.; Gulder, T.; Gulder, T.A.M. Total synthesis of axially chiral biaryl natural products. Chem. Rev. 2011, 111(2), 563-639. doi:10.1021/cr100155e.

46. Pouysegu, L.; Deffieux, D.; Gaelle, G.M. Synthesis of ellagitannin natural products. Nat. Prod. Rep. 2011, 28(5), 853-874. doi:10.1039/c0np00058b.

47. Snyder, S.A.; Gollner, A.; Chiriac, M.I. Regioselective reactions for programmable resveratrol oligomer synthesis. Nature, 2011, 474(7352): 461-466. doi:10.1038/nature10197.

48. Quideau, S. Organic chemistry: Triumph for unnatural synthesis. Nature, 2011, 474 (7352): 459-460.

49. Osman, A.M.; Wong, K.K.Y.; Fernyhough, A. ABTS radical-driven oxidation of polyphenols: Isolation and structural elucidation of covalent adducts. Biochemical and Biophysical Research Communications, 2006, 346(1): 321-329. doi:10.1016/j.bbrc.2006.05.118.

50. Papadopoulou, A.; Frazier, R.A. Characterization of protein-polyphenol interactions. Trends in Food Sci $\mathcal{E}$ Technol. 2004, 15(3-4): 186-90. doi:10.1016/j.tifs.2003.09.017.

51. Jindal K.K.; Sharma R.C. Recent trends in horticulture in the Himalayas. Indus Publishing, 2004, ISBN 978-817387-162-7.

52. Aizpurua-Olaizola, O.; Ormazabal, M.; Vallejo, A.; Olivares, M.; Navarro, P.; Etxebarria, N.; Usobiaga, A. Optimization of supercritical fluid consecutive extractions of fatty acids and polyphenols from vitis vinifera grape wastes. Journal of Food Science. 2015, 80(1), E101-7.

53. Lattanzio V. Role of phenolics in the resistance mechanisms of plants against fungal pathogens and insects (and references therein). Phytochemistry: Advances in Research, 2006, 23-67. 
54. Hättenschwiler, S.; Vitousek, P.M. The role of polyphenols in terrestrial ecosystem nutrient cycling. Trends in Ecology E Evolution, 2000, 15(6), 238-243. doi:10.1016/S0169-5347(00)01861-9.

55. Popa, V.; Dumitru, M.; Volf, I.; Anghel, N. Lignin and polyphenols as allelochemicals. Industrial Crops and Products, 2008, 27(2), 144-9. doi:10.1016/j.indcrop.2007.07.019

56. Tanner, G.J.; Francki, K.T.; Abrahams, S.; Watson, J.M.; Larkin, P.J.; Ashton, A.R. Proanthocyanidin biosynthesis in plants. Journal of Biological Chemistry, 2003, 278(34), 31647-56.

57. Krasnow, M.N.; Murphy, T. M. Polyphenol glucosylating activity in cell suspensions of grape (Vitis vinifera). Journal of Agricultural and Food Chemistry, 2004, 52(11), 3467-3472.

58. Hertweck, C. The Biosynthetic Logic of Polyketide Diversity. Angewandte Chemie International Edition, 2009, 48(26): 4688-4716. doi:10.1002/anie.200806121.

59. d'Archivio, M.; Filesi, C.; Varì, R.; Scazzocchio, B.; Masella, R. Bioavailability of the polyphenols: status and controversies. International Journal of Molecular Sciences, 2010, 11(4), 1321-1342.

60. Mennen L. Risks and safety of polyphenol consumption. Am J Clin Nutr, 2005, 81(1), 3265-3295.

61. Gross, P. New Roles for Polyphenols. A 3-Part Report on Current Regulations and the State of Science. Nutraceuticals World, 2009.

62. US Food and Drug Administration (2017). "Guidance for Industry: Food Labeling; Nutrient Content Claims; Definition for "High Potency" and Definition for "Antioxidant" for Use in Nutrient Content Claims for Dietary Supplements and Conventional Foods; Small Entity Compliance Guide". Center for Food Safety and Applied Nutrition, US Food and Drug Administration.

63. Halliwell, B. Dietary polyphenols: Good, bad, or indifferent for your health? Cardiovasc Res. 2007, 73(2), 341347. doi:10.1016/j.cardiores.2006.10.004.

64. EFSA. Scientific Opinion on the substantiation of health claims related to: flavonoids and ascorbic acid in fruit juices, including berry juices (ID 1186); flavonoids from citrus (ID 1471); flavonoids from Citrus paradisi Macfad. (ID 3324, 3325); flavonoids (ID. EFSA Journal, 2011, 9(4): 2082.

65. EFSA. Scientific Opinion on the modification of the authorisation of a health claim related to cocoa flavanols and maintenance of normal endothelium-dependent vasodilation pursuant to Article 13(5) of Regulation (EC) No 1924/2006 following a request in accordance with Article 19 of Regulation (EC) No 1924/2006. EFSA Journal, 2014, 12(5).

66. Haslam, E.; Lilley, T.H.; Cai, Y.; Martin, R.; Magnolato, D. Traditional herbal medicines--the role of polyphenols. Planta Med. 1989, 55(1), 1-8. doi:10.1055/s-2006-961764.

67. Lesschaeve I.; Noble, A.C. Polyphenols: factors influencing their sensory properties and their effects on food and beverage preferences. Am J Clin Nutr. 2005, 81(1), 330S-335S.

68. Hufnagel, J.C.; Hofmann, T. Orosensory-directed identification of astringent mouthfeel and bitter-tasting compounds in red wine. J Agric Food Chem. 2008, 56(4), 1376-1386. doi:10.1021/jf073031n.

69. Pan, X. Microwave-assisted extraction of tea polyphenols and tea caffeine from green tea leaves. Chemical Engineering and Processing. 2003, 42(2), 129-33. doi:10.1016/S0255-2701(02)00037-5.

70. Arranz, S.; Saura-Calixto, F.; Shaha, S.; Kroon, P.A. High contents of nonextractable polyphenols in fruits suggest that polyphenol contents of plant foods have been underestimated. Journal of Agricultural and Food Chemistry. 2009, 57(16), 7298-303. doi:10.1021/jf9016652. hdl:10261/82508.

71. Owen, R.W.; Haubner, R.; Hull, W.E.; Erben, G.; Spiegelhalder, B.; Bartsch, H.; Haber, B. Isolation and structure elucidation of the major individual polyphenols in carob fibre. Food and Chemical Toxicology, 2003, 41(12), 1727-38. doi:10.1016/S0278-6915(03)00200-X.

72. Walker, R.B.; Everette, J.D. Comparative reaction rates of various antioxidants with ABTS radical cation. Journal of Agricultural and Food Chemistry, 2009, 57(4), 1156-1161.

73. Pulido, R.; Bravo, L.; Saura-Calixto, F. Antioxidant activity of dietary polyphenols as determined by a modified ferric reducing/antioxidant power assay. Journal of Agricultural and Food Chemistry, 2000, 48(8), 33963402.

74. Mello, L.; Sotomayor, M.D.P.T.; Kubota, L.T. HRP-based amperometric biosensor for the polyphenol's determination in vegetables extract. Sensors and Actuators B: Chemical, 2003, 96(3), 636-645.

75. Eberhard, B. Terpenes: Flavors, Fragrances, Pharmaca, Pheromones. Wiley-VCH. 2006. 
76. Davis, E.M.; Croteau, R. Cyclization enzymes in the biosynthesis of monoterpenes, sesquiterpenes, and diterpenes. Topics in Current Chemistry, 2000, 209, 53-95.

77. Martin, D.M.; Gershenzon, J.; Bohlmann, J. Induction of volatile terpene biosynthesis and diurnal emission by methyl jasmonate in foliage of norway spruce. Plant Physiology, 2003, 132(3), 1586-1599.

78. Pichersky, E. Biosynthesis of plant volatiles: nature's diversity and ingenuity. Science, 2006, 311(5762), 808811.

79. Kumari, I.; Ahmed, M.; Akhter, Y. Evolution of catalytic microenvironment governs substrate and product diversity in trichodiene synthase and other terpene fold enzymes. Biochimie, 2017, 144, 9-20.

80. Pazouki, L.; Niinemets, Ü. multi-substrate terpene synthases: their occurrence and physiological significance. Frontiers in Plant Science, 2016, 7, 10-19. doi:10.3389/fpls.2016.01019.

81. Breitmaier, E. Terpenes: Flavors, Fragrances, Pharmaca, Pheromones. John Wiley \& Sons. 2006, pp. 1-13.

82. Zelena, K.; Hardebusch, B.; Hülsdau, B.; Berger, R.G.; Zorn, H. Generation of norisoprenoid flavors from carotenoids by fungal peroxidases. Journal of Agricultural and Food Chemistry, 2009, 57(21), 99519955. doi:10.1021/jf901438m.

83. Thimmappa, R.; Geisler, K.; Louveau, T.; O'Maille, P.; Osbourn, A. Triterpene biosynthesis in plants. Annual Review of Plant Biology, 2014, 65, 225-257.

84. Adam, D. Scientists discover cloud-thickening chemicals in trees that could offer a new weapon in the fight against global warming". The Guardian. 2008.

85. Steenackers, B.; De Cooman, L.; De-Vos, D. Chemical transformations of characteristic hop secondary metabolites in relation to beer properties and the brewing process: A review. Food Chemistry, 2015, 172, 742756.

86. Boutanaev, A.M.; Moses, T.; Zi, J.; Nelson, D.R.; Mugford, S.T.; Peters, R.J.; Osbourn, A. Investigation of terpene diversification across multiple sequenced plant genomes. Proceedings of the National Academy of Sciences, 2015, 112(1), E81-E88. doi:10.1073/pnas.1419547112.

87. André, C.M.; Hausman, J.F.; Guerriero, G. Cannabis sativa: The plant of the thousand and one molecules. Frontiers in Plant Science, 2016, 7, 19. doi:10.3389/fpls.2016.00019

88. Booth, J.K.; Page, J.E.; Bohlmann, örg.; Hamberger, Björn (ed.). Terpene synthases from Cannabis sativa. PLOS ONE, 2017, 12(3), e0173911. doi:10.1371/journal.pone.0173911. ISSN 1932-6203.

89. Brito-Arias, M. Synthesis and Characterization of Glycosides. Springer, 2007.

90. IUPAC. "Glycosides". IUPAC Gold Book-Glycosides. 2009

91. Lindhorst, T.K. Essentials of Carbohydrate Chemistry and Biochemistry. Wiley-VCH, 2007.

92. Gleadow, R.M.; Møller, B.L. Cyanogenic glycosides: synthesis, physiology, and phenotypic plasticity. Annual Review of Plant Biology, 2014, 65, 155-85.

93. Milazzo, S.; Horneber, M. Laetrile treatment for cancer. The Cochrane Database of Systematic Reviews, 2015, 4, CD005476.

94. Sun, H.X.; Xie, Y.; Ye, Y.P. Advances in saponin-based adjuvants. Vaccine. 2009, 27(12), 1787-1796.

95. Patra, A.K.; Saxena, J. The effect and mode of action of saponins on the microbial populations and fermentation in the rumen and ruminant production. Nutrition Research Reviews. 2009, 22(2), 204-209.

(C) 2019 by the authors. Submitted for possible open access publication under the terms and conditions of the Creative Commons Attribution (CC BY) license (http://creativecommons.org/licenses/by/4.0/). 